\title{
A vector polarimeter at MAMI
}

\author{
Fabian Nillius ${ }^{* \dagger}$ \\ Institut fuer Kernphysik \\ Johannes Gutenberg-Universitaet, Mainz. \\ E-mail: nilliusdkph.uni-mainz.de \\ Kurt Aulenbacher \\ Institut fuer Kernphysik \\ Johannes Gutenberg-Universitaet, Mainz \\ E-mail: aulenbacekph.uni-mainz.de
}

\begin{abstract}
Atomic bremsstrahlung-polarisation correlation between incoming electrons and outgoing photons may allow to design a polarimeter capable of measuring all components of beam polarisation simultaneously. This device can be called a vector polarimeter. It might allow to test theoretical predictions of correlation coefficients between 1 and $3.5 \mathrm{MeV}$ as well as work as a beam diagnostics device. As a first step we have set up a measurement of the helicity transfer to the photon as a function of electron energy based on the Compton absorption method.
\end{abstract}

XVIth International Workshop on Polarized Sources, Targets, and Polarimetry, PSTP2015,

14-18 September 2015,

Bochum, Germany

*Speaker.

${ }^{\dagger}$ This work was supported throughout by BMBF Verbundforschung within project 05H12UM6 "Spin Optimierung" 


\section{Introduction}

\subsection{The MAMI accelerator}

The MAInZER MiKROtron is a cascade of microtrons to accelerate electrons up to $1.6 \mathrm{GeV}$. Equipped with a polarised gun, it can provide a highly polarised beam with an adjustable polarisation vector, which is achieved by a Wien filter based spin rotator [四]. To detect the degree of polarisation, a polarimeter was installed using Mott [[]] scattering in an extraction beamline behind the injector linac, which is operated at an energy of $3.5 \mathrm{MeV}$. This Mott polarimeter is only sensitive to the component of the polarisation vector which is perpendicular to the electron momentum and lies within the accelerator plane. A vector polarimeter would be able to detect more components of the polarisation vector simultaneously.

\subsection{The correlation tensor}

Tseng and Pratt [3] introduced a tensor $C_{\mu \nu}(\mathrm{Z}, \vartheta, \mathrm{E})$ which describes the correlation between the incoming electron polarisation and the polarisation of the emitted photons. For the purpose of the presented vector polarimeter, we use the outgoing circularly polarised bremsstrahlung. This is why only the entries of the correlation tensor with $v=2$ are of interest.

For right-hand circularly polarised photons, the cross section can be parameterised according to

$$
\frac{\mathrm{d}^{2} \sigma}{\mathrm{d} \omega \mathrm{d} \Omega_{\mathrm{k}}}=C_{0}\left(1-\eta_{x} C_{12}-\eta_{y} C_{20}-\eta_{z} C_{32}\right)
$$

where $\eta_{x}, \eta_{y}, \eta_{z}$ are the cartesian components of the spin vector of the electron beam ${ }^{1}$ and $C_{0}$ is a spin-independent cross section.

\subsection{Measuring principle of the Compton absorption polarimeter}

The polarised electron produces circularly polarised photons. To detect the outgoing circular polarisation, the bremsstrahlung passes a magnetised absorber. We use the fact that the Compton cross section [四] has a spin-dependent part. The bremsstrahlung scatters on the polarised electrons inside the magnetic absorber and is therefore scattered out of the detector volume. This design is also known as a Compton absorption polarimeter [[]].

For a completely circularly polarised photon beam passing a completely polarised absorber, the asymmetry can be written like ㄴ.]:

$$
A_{\text {full }}\left(E_{\gamma}\right)=\frac{e^{-\sigma_{p} Z N_{\mathrm{A}} \rho d / B}-e^{+\sigma_{p} Z N_{\mathrm{A}} \rho d / B}}{e^{-\sigma_{p} Z N_{\mathrm{A}} \rho d / B}+e^{+\sigma_{p} Z N_{\mathrm{A}} \rho d / B}}=-\tanh \left(\frac{\sigma_{p} Z N_{\mathrm{A}} \rho d}{B}\right)
$$

with $\sigma_{p}=$ spin-dependent Compton cross section, $Z=$ atomic number of the absorber, $B=$ baryon number of the absorbing material, $N_{\mathrm{A}}=$ Avogadro number, $d=$ absorber length and $\rho=$ density of the absorber. Taking into account that the electron beam is not completely polarised and the fact that the entry of the correlation tensor $C_{32}\left(E_{\gamma}, \theta, Z\right)$ is a function of energy equation $\square$ transforms into $\mathbb{L 2}$ and finally $\mathbb{\square . 3}$ for a small argument of the tanh. The atomic number $Z$ is replaced by 2

\footnotetext{
${ }^{1} \mathrm{z}$-axis pointing in beam direction and y-axis is perpendicular to the photon emission plane.
} 
because only approximately two electrons contribute to the magnetisation inside the absorber. The degree of polarisation of the electron beam is $85 \%$.

$$
\begin{aligned}
A\left(E_{\gamma}\right) & =-P_{e} C_{32}\left(E_{\gamma}\right) \tanh \left(\sigma_{p}\left(E_{\gamma}\right) \frac{2 \mathrm{~N}_{A} \rho d}{B}\right) \\
& \approx-P_{e} C_{32}\left(E_{\gamma}\right) \sigma_{p}\left(E_{\gamma}\right) \frac{2 N_{A} \rho d}{B}
\end{aligned}
$$

with $\quad P_{e}=$ polarisation of the electron beam

\section{Setup and measurements}

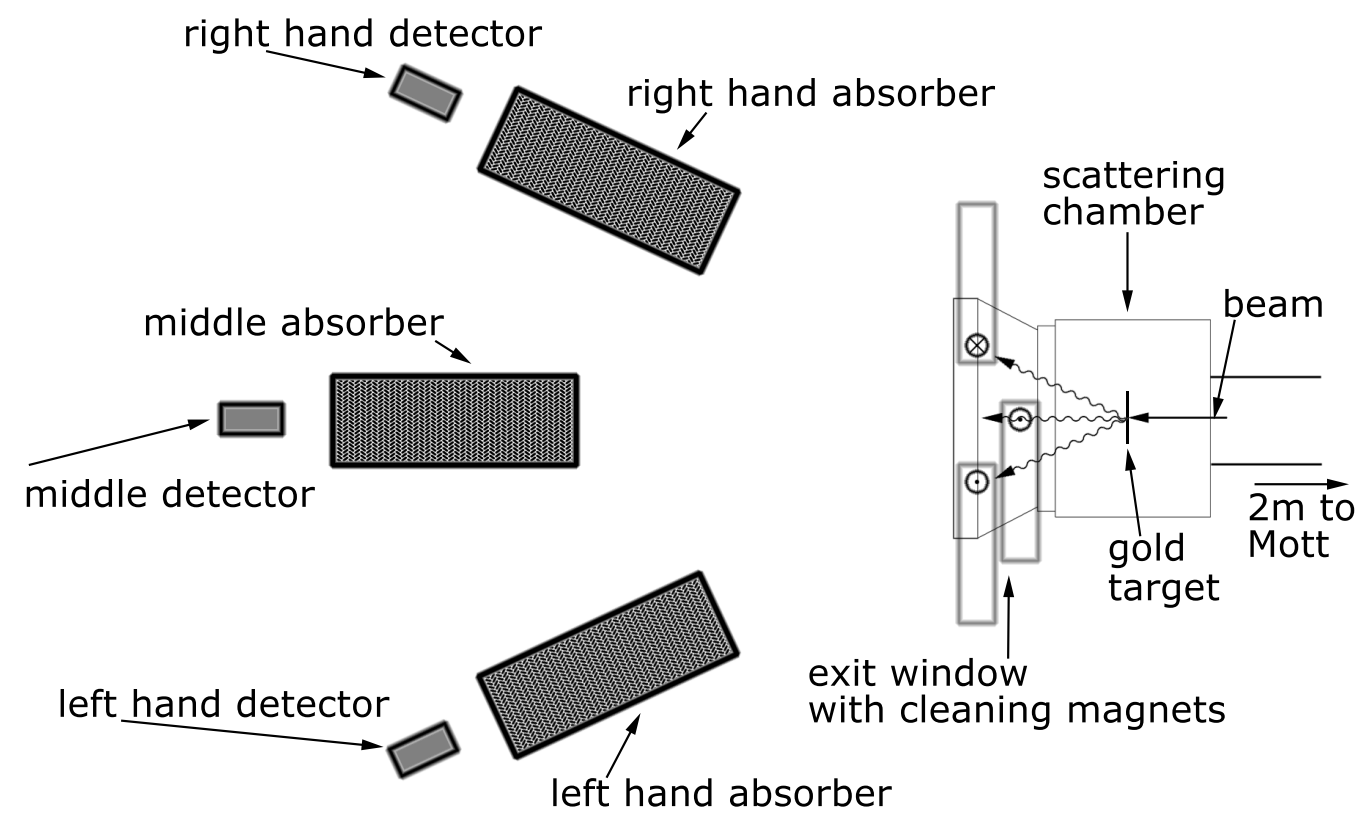

Figure 1: Experimental setup

Figure 1 shows a schematic sketch of the experimental setup. The polarised electron beam with an energy of $3.5 \mathrm{MeV}$ is coming from the right and produces bremsstrahlung at the gold target. Magnets apply magnetic fields over the trapezoidal exit window to deflect the scattered electrons in order to have only the bremsstrahlung photons traveling towards the magnetic absorbers and detectors. The magnetic absorbers are excited by solenoid coils to polarise the electrons inside the material. Behind the absorbers the $\gamma$ radiation is detected by LYSO crystals with photomultiplier tubes. The signals are passed to multi channel analysers which are an in-house development of the electronic department for making a histogram of the photon spectra.

\subsection{Energy resolved measurement of the transverse polarisation via $C_{12}\left(\theta \neq 0, E_{\gamma}\right)$ and} $C_{32}\left(\theta \neq 0, E_{\gamma}\right)$

For these measurements, the detectors to the left and the right are used since $C_{12}(\theta=0)=0$ as in [目]. It describes the correlation between the transverse polarisation of the electrons within the 
accelerator plane and the circular polarisation of the outgoing photon. But $C_{32}$ contributes to the circularly polarised photons aswell. This is why the asymmetry should follow function 2.1$]$

$$
A\left(\phi_{\mathrm{Pol}}\right)=\chi\left(C_{32} \cos \left(\phi_{\mathrm{Pol}}\right) \pm C_{12} \sin \left(\phi_{\mathrm{Pol}}\right)\right)
$$

where $\chi$ is a constant of proportionality. Since $C_{12}$ has a change in sign from the left to right side, the function is symmetric again. Figure 3 shows the results of the measurement of asymmetry as a function of the polarisation angle $\phi_{\mathrm{Pol}}$ for different photon energies. The plus sign in $2.1 \mathrm{l}$ is for the right hand detector and the minus sign for the left hand detector. The solid lines in the plot come from fits to equation 1 . The predicted contribution due to a non-zero value of $C_{12}$ is clearly visible from the data.

The value at $\phi_{\mathrm{Pol}}=0^{\circ}$ gives $C_{32}$ while from the values at $\phi_{\mathrm{Pol}}= \pm 90^{\circ}$ it is possible to calculate $C_{12}$. The extracted values of $C_{12}$ are in agreement with the theory within the limits of the statistical errors. The systematic errors are under investigation.
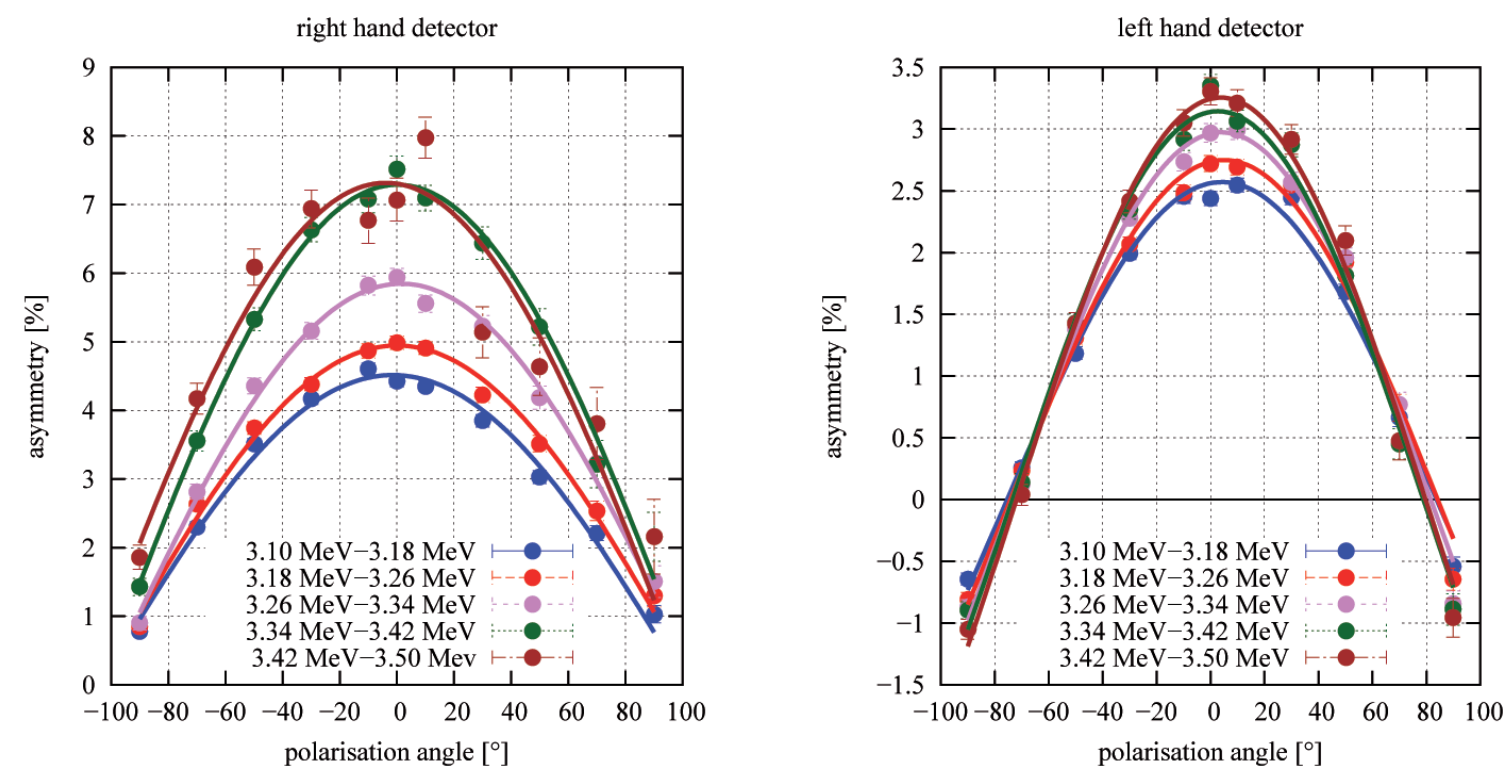

Figure 2: Asymmetry in left hand detector and right hand detector as a function of photon energy for $\theta=21^{\circ}$

\section{Summary \& Outlook}

We have performed measurements of photon transmission through magnetic absorbers under $\theta=21^{\circ}$ to gain access to $C_{32}\left(E_{\gamma}, \theta\right)$ and $C_{12}\left(E_{\gamma}, \theta\right)$. To our knowledge, this is the first time that the effect of transverse polarization being transferred into circular photon polarisation has been observed in this energy range. The knowledge of such correlation coefficients is helpful for the exact modelling of spin transport in thick targets, such as the polarised positron target for ILC. The values are important for the modeling of spin transport and will be used for further projects, for instance polarised positron sources. Moreover, this apparatus can measure different components of the polarisation vector at the same time, a so-called vector polarimeter. 


\section{References}

[1] V. Tioukine and K. Aulenbacher, Operation of the MAMI accelerator with a Wien filter based spin rotation system, Nuclear Instruments and Methods in Physics Research Section A: Accelerators, Spectrometers, Detectors and Associated Equipment 568 (2006), no. 2537 - 542.

[2] V. Tioukine, K. Aulenbacher, and E. Riehn, A Mott polarimeter operating at MeV electron beam energies, Review of Scientific Instruments 82 (2011), no. 3033303.

[3] H. K. Tseng and R. H. Pratt, Polarization correlations in atomic-field bremsstrahlung, Phys. Rev. A 7 (May, 1973) 1502-1515.

[4] O. Klein and Y. Nishina, Über die Streuung von Strahlung durch freie Elektronen nach der neuen relativistischen Quantendynamik von Dirac, Zeitschrift für Physik 52 (1929), no. 11-12 853-868.

[5] H. Schopper, Measurement of circular polarization of $x$-rays, Nuclear Instruments 3 (1958), no. 3158 $-176$.

[6] D. Jakubassa-Amundsen, Polarization transfer in relativistic electron-nucleus bremsstrahlung, Physics Letters A 375 (2011), no. 151671 - 1673. 